\title{
Affine surfaces with parallel shape operators
}

\author{
by WŁodzimierz JeloneK (Kraków)
}

\begin{abstract}
We study affine nondegenerate Blaschke hypersurfaces whose shape operators are parallel with respect to the induced Blaschke connections. We classify such surfaces and thus give an exact classification of extremal locally symmetric surfaces, first described by F. Dillen.
\end{abstract}

1. Introduction. There are several results concerning the affine hypersurfaces whose cubic forms $C$ are parallel with respect to the affine connection $\nabla$ or to $\nabla^{\wedge}$ (the Levi-Cività connection of the affine metric). Amongst others this problem was investigated by M. Magid, K. Nomizu and U. Pinkall (see for example [3], [6]). The aim of our paper is to characterize affine hypersurfaces with an equiaffine structure $(\nabla, \xi)$ whose shape operator is parallel with respect to $\nabla$ or $\nabla^{\wedge}$. In particular, we classify such surfaces in the case when $(\nabla, \xi)$ is a Blaschke structure .

2. Notations. Let $M$ be a smooth, oriented and connected manifold, $V$ a real vector space with a fixed orientation form $o, \operatorname{dim} V=\operatorname{dim} M+1$. When $V=\mathbb{R}^{n+1}$ we assume $o=\operatorname{det}$, where det is the standard orientation form on $\mathbb{R}^{n+1}$. Let $f: M \rightarrow V$ be an immersion. Any subbundle $\Xi$ of $f^{*} T V=M \times V$ transversal to $f_{*}(T M)$ (i.e. $f^{*} T V=f_{*}(T M) \oplus \Xi$ ) defines an affine connection in the following way $\left(f_{*}(X):=d f(X)\right)$ :

$$
\pi\left(D_{X} f_{*} Y\right)=f_{*}\left(\nabla_{X} Y\right)
$$

where we denote by $D$ the standard flat connection on any vector space and $\pi$ is the projection $\pi: f^{*} T V \rightarrow f_{*}(T M)$ parallel to $\Xi$. If $\xi$ is a nowhere vanishing (n.v.) section of $\Xi$ then it defines a symmetric form $g$ by

$$
D_{X}\left(f_{*} Y\right)=f_{*}\left(\nabla_{X} Y\right)+g(X, Y) \xi .
$$

1991 Mathematics Subject Classification: Primary 53A15.

Key words and phrases: Blaschke structure, affine locally symmetric surface, affine shape operator, equiaffine structure, affine normal field. 
An immersion $f$ is called nondegenerate if $g$ is a nondegenerate form. In the rest of our paper we assume this condition for $f$. $\xi$ defines also the shape operator $S$ and the transversal connection form $\tau$ by (see [5])

$$
D_{X} \xi=-f_{*}(S X)+\tau(X) \xi .
$$

With $\xi$ there is associated the volume element $\nu_{\xi}=f^{*}\left(i_{\xi} o\right)$. The connection $\nabla$ is called equiaffine if there exists an n.v. section $\xi$ of $\Xi$ for which $\tau=0$ (this means $\nabla \nu_{\xi}=0$ ); $\xi$ is then called an equiaffine normal field. For a given equiaffine connection an equiaffine normal field is uniquely determined up to a constant factor. An equiaffine structure $(\nabla, \xi)$ on a hypersurface $(M, f)$ is called a Blaschke structure when $\nu_{\xi}=\nu_{g}$, where $\nu_{g}$ is the volume element of the nondegenerate metric $g$; $\xi$ is then called an affine normal field (see [4], [5]). For a hypersurface $(M, f)$ with an equiaffine structure $(\nabla, \xi)$ the following equations hold:

$$
\begin{gathered}
\nabla S(X, Y)=\nabla S(Y, X), \\
g(X, S Y)=g(S X, Y) .
\end{gathered}
$$

A hypersurface with an equiaffine structure is called an equiaffine sphere if $S=\lambda \operatorname{Id}_{T M}$. In that case from (C) it follows that $\lambda$ is constant.

The conormal field $\xi^{\vee}: M \rightarrow V^{*}$ is defined by the conditions

$$
\left\langle\xi^{\vee}, f_{*}(T M)\right\rangle=0, \quad\left\langle\xi^{\vee}, \xi\right\rangle=1,
$$

where $\langle\rangle:, V^{*} \times V \rightarrow \mathbb{R}$ is the standard bilinear form $\langle\phi, v\rangle:=\phi(v) . \xi^{\vee}$ is an immersion whenever $f$ is a nondegenerate immersion, and defines a connection $\nabla^{\vee}$ by

$$
D_{X}\left(\xi_{*}^{\vee} Y\right)=\left(\xi^{\vee}\right)_{*}\left(\nabla_{X}^{\vee} Y\right)-B(X, Y) \xi^{\vee} \quad(B(X, Y)=g(X, S Y)) .
$$

Let $\nabla^{\wedge}$ be the Levi-Cività connection for $g$ and let $(\nabla, \xi)$ be an equiaffine structure. Then

$$
\begin{gathered}
\nabla^{\wedge}=\frac{1}{2}\left(\nabla+\nabla^{\vee}\right), \\
\nabla=\nabla^{\wedge}+K, \quad \nabla^{\vee}=\nabla^{\wedge}-K,
\end{gathered}
$$

where $K$ is a symmetric $(1,2)$-tensor. We also have

$$
C(X, Y, Z)=\nabla g(X, Y, Z)=-2 g(K(X, Y), Z) \text {. }
$$

$C$ is a symmetric form called the cubic form. A Blaschke structure is characterized by the apolarity condition:

$$
\sum g^{i j} C\left(X, \partial_{i}, \partial_{j}\right)=0 .
$$

Denote by $R$ the curvature tensor for $\nabla$. Then

$$
R(X, Y) Z=g(Y, Z) S X-g(X, Z) S Y .
$$


3. The shape operator and the curvature tensor. Now we consider the equation $\nabla S=0$.

Proposition 1. Let $(M, f)$ be a nondegenerate hypersurface with an equiaffine structure $(\nabla, \xi)$. Then the following conditions are equivalent:
(a) $\nabla S=0$.
(b) $\nabla^{\wedge} S=0$.
(c) $\nabla^{\vee} S=0$.

Proof. From (2) it follows that

(5) $\quad \nabla S(X, Y)=\nabla^{\wedge} S(X, Y)-S K(X, Y)+K(X, S Y)$,

(6) $\quad \nabla^{\vee} S(X, Y)=\nabla^{\wedge} S(X, Y)+S K(X, Y)-K(X, S Y)$.

(b) $\Rightarrow\left(\right.$ a), (c). Let $\nabla^{\wedge} S=0$. Then from (5) and (C) it follows that $-S K(X, Y)+K(X, S Y)=-S K(Y, X)+K(Y, S X)$.

As $K$ is symmetric we obtain

$$
K(X, S Y)=K(S X, Y)
$$

From (3) we then have

$$
\nabla g(S X, Y, Z)=\nabla g(X, S Y, Z)=\nabla g(X, Y, S Z)
$$

as $\nabla g$ is symmetric. Hence using (R) and (3) we get

$$
g(K(S X, Y), Z)=g(K(X, Y), S Z)=g(S K(X, Y), Z)
$$

for any $X, Y, Z$. As $g$ is nondegenerate this means $K(S X, Y)=S K(X, Y)$ and from (5), (6) we obtain $\nabla S=0, \nabla^{\vee} S=0$.

(a) $\Rightarrow(\mathrm{b})$. From (R) and $\nabla S=0$ we have

$$
\nabla g(X, S Y, Z)=\nabla g(X, Y, S Z)
$$

for any $X, Y, Z$, which implies $K(X, S Y)=S K(X, Y)$ as above. Hence from (6) we get $\nabla^{\wedge} S=0$.

In a similar way one can prove $(\mathrm{c}) \Rightarrow$ (b) in view of the equality $\nabla g=$ $-\nabla^{\vee} g$, which follows from (1) and $\nabla^{\wedge} g=0$.

Remark. Note that the equivalence $\nabla S=0 \Leftrightarrow \nabla^{\wedge} S=0$ distinguishes the shape operator from other affine tensors. For example the equivalence $\nabla C=0 \Leftrightarrow \nabla^{\wedge} C=0$ does not hold (see [3], [6]).

THEOREM 1. Let $(M, f)$ be a nondegenerate hypersurface with an equiaffine structure $(\nabla, \xi)$ and let $S$ be the shape operator. Then the following conditions are equivalent:

(a) R.S $=0$ where $R$ acts on $S$ as a derivation, i.e.

$$
(R(X, Y) . S) Z=R(X, Y)(S Z)-S(R(X, Y) Z)=0 .
$$

(b) Either $S=\lambda \operatorname{Id}_{T M}$ or $\left(S^{2}=0\right.$ and $\left.\operatorname{rank} S \leq 1\right)$. 
Proof. Fix $x_{0} \in M$. Denote $S_{x_{0}}$ also by $S$. Condition (a) is equivalent to

$$
g(Y, Z) S^{2} X-g(X, Z) S^{2} Y=g(S Y, Z) S X-g(S X, Z) S Y .
$$

Let $\lambda, \mu \in \mathbb{C}$ be two eigenvalues of $S$ and let $X, Y \in T_{x_{0}}^{c} M$ be eigenvectors of $S, S X=\lambda X, S Y=\mu Y$. From (8) we obtain

$$
\left(\lambda^{2}-\mu \lambda\right) g(Y, Z) X=\left(\mu^{2}-\mu \lambda\right) g(X, Z) Y
$$

for every $Z$. It follows that $\lambda^{2}-\mu \lambda=0, \mu^{2}-\mu \lambda=0$, which implies $\mu=\lambda$. Therefore $S$ has exactly one real eigenvalue.

It follows that either $S=\lambda I$ or there exist two nonzero vectors $X, Y \in$ $T_{x_{0}} M$ such that $S X=\lambda X$ and $S Y=\lambda Y+X$. From (8) we then obtain

$$
g(Y, Z) \lambda^{2} X-g(X, Z)\left(\lambda^{2} Y+2 \lambda X\right)=g(Y, Z) \lambda^{2} X-g(X, Z) \lambda^{2} Y,
$$

which means that $\lambda g(X, Z) X=0$ for all $Z$, and thus $\lambda=0$. If we take in the equation (8) $X$ as above then we obtain

$$
-g(X, Z) S^{2} Y=0
$$

for all $Z, Y$, hence $S^{2}=0$. This implies that in that case (a) is equivalent to $g(S Y, Z) S X=g(S X, Z) S Y$. Hence for all $X, Y, S X$ and $S Y$ are linearly dependent. It follows that $\operatorname{rank} S \leq 1$.

As $M$ is connected, assuming condition (a), if there exists a point $x_{0}$ such that $S_{x_{0}}=\lambda I$ and $\lambda \neq 0$ then $S=\lambda I$ on the whole of $M$. Otherwise $S^{2}=0$ and $\operatorname{rank} S \leq 1$ on $M$.

Corollary 1. Let $(M, f)$ be a nondegenerate hypersurface with an equiaffine structure $(\nabla, \xi)$ and a parallel shape operator. Then either $(M, f)$ is an equiaffine sphere or $\left(S^{2}=0\right.$ and $\left.\operatorname{rank} S=1\right)$.

COROLlary 2. A locally strongly convex hypersurface $(M, f)$ with an equiaffine structure $(\nabla, \xi)$ is an equiaffine sphere if and only if its shape operator is $\nabla$-parallel.

Re mark. When $\operatorname{rank} S>1$ we do not have to assume that $(\nabla, \xi)$ is an equiaffine structure to deduce Corollary 1 . We show that an affine structure $(\nabla, \xi)$ such that $\nabla S=0$ and $\operatorname{rank} S>1$ is an equiaffine structure, i.e. $\tau=0$. For an affine structure $(\nabla, \xi)$ the Codazzi equation has the form (see [5])

$$
\nabla S(X, Y)-\nabla S(Y, X)=\tau(X) S Y-\tau(Y) S X
$$

If $S X=0$ then take $Y$ such that $S Y \neq 0$. From $(\mathrm{C} 1)$ we obtain $\tau(X)=$ 0 . Let now $X$ be a vector for which $S X \neq 0$. Since rank $S>1$ there exists $Y$ such that $S X$ and $S Y$ are linearly independent. (C1) implies that $\tau(X) S Y=\tau(Y) S X$, which means $\tau(X)=0$ also in that case and proves the equality $\tau=0$. 
COROLlary 3. Let $(M, f)$ be a nondegenerate hypersurface with an affine structure $(\nabla, \xi)$ for which the shape operator is $\nabla$-parallel. If there exists $x_{0} \in M$ such that $\operatorname{rank} S_{x_{0}}>1$ then $(\nabla, \xi)$ is an equiaffine structure and $S=\lambda I$.

4. The classification theorem. F. Dillen [1] obtained examples of surfaces with locally symmetric Blaschke connections and parallel shape operators. These examples are given by

$$
\begin{aligned}
& x(u, v)=(u \cos (v)+f(v), u \sin (v)+g(v), v), \\
& x(u, v)=(u \cosh (v)+f(v), u \sinh (v)+g(v), v) .
\end{aligned}
$$

Note that the examples are not in one-to-one correspondence with pairs $(f, g)$ of smooth functions; for example, $(f, g)$ and $(f+h \cos , g+h \sin )$ give the same surface. Each of these examples is affinely equivalent to one of the surfaces $\left(\mathbb{R}^{2}, f_{i, \phi}\right)$ given below, which are special cases of Dillen's examples.

Let $\phi \in C^{\infty}(\mathbb{R})$ be any smooth function. Set

$$
\begin{array}{ll}
v_{1}(x):=(\cos x,-\sin x, 0), & V_{1}(x):=(\sin x, \cos x, 0), \\
v_{2}(x):=(\cosh x, \sinh x, 0), & V_{2}(x):=(\sinh x, \cosh x, 0) .
\end{array}
$$

Then we have

$$
V_{i}^{\prime}=v_{i}, \quad i=1,2 \quad\left(f^{\prime}:=d f / d x\right) .
$$

Let $w_{i}:=\left(w_{i}^{1}, w_{i}^{2}, 0\right)=w_{i}^{1} e_{1}+w_{i}^{2} e_{2}$ satisfy the equation

$$
w_{i}^{\prime \prime}=\phi v_{i}-V_{i}
$$

and define $u_{i}:=w_{i}-x e_{3}$. Set $\varepsilon_{1}=1, \varepsilon_{2}=-1$.

If we define $f_{i, \phi}: \mathbb{R}^{2} \rightarrow \mathbb{R}^{3}$ by

$$
f_{i, \phi}\left(x^{1}, x^{2}\right):=u_{i}\left(x^{1}\right)+x^{2} v_{i}\left(x^{1}\right)
$$

then

$$
\xi_{i, \phi}\left(x^{1}, x^{2}\right):=-V_{i}\left(x^{1}\right)
$$

is an affine normal field for $\left(\mathbb{R}^{2}, f_{i, \phi}\right)$ and it is easy to check directly the equalities

$$
\begin{gathered}
\Gamma_{11}^{2}=-\varepsilon_{i} x^{2}+\phi\left(x^{1}\right), \quad \Gamma_{j k}^{i}=0 \quad \text { for other } i, j, k, \\
S \partial_{1}=\partial_{2}, \quad S \partial_{2}=0, \\
g\left(\partial_{1}, \partial_{1}\right)=1, \quad g\left(\partial_{1}, \partial_{2}\right)=\varepsilon_{i}, \quad g\left(\partial_{2}, \partial_{2}\right)=0,
\end{gathered}
$$

in the standard coordinates on $\mathbb{R}^{2}$. $f_{i, \phi}$ is not uniquely determined by $\phi$ (by virtue of (9)). In particular, for $\phi=0$ we can take as $f_{i, 0}$ the immersion $f_{i}$ :

$$
\begin{aligned}
& f_{1}\left(x^{1}, x^{2}\right):=\left(\sin x^{1}+x^{2} \cos x^{1}, \cos x^{1}-x^{2} \sin x^{1},-x^{1}\right), \\
& f_{2}\left(x^{1}, x^{2}\right):=\left(-\sinh x^{1}+x^{2} \cosh x^{1},-\cosh x^{1}+x^{2} \sinh x^{1},-x^{1}\right) .
\end{aligned}
$$


LEMMA. Let $S$ be a parallel shape operator on an affine surface $(M, f)$, $\operatorname{dim} M=2$, such that $S^{2}=0$ and $\operatorname{rank} S=1$. Then around any $x_{0} \in M$ there exists a local chart on $M$ with canonical basis $\left\{\partial_{1}=\partial / \partial x^{1}, \partial_{2}=\right.$ $\left.\partial / \partial x^{2}\right\}$ for which

$$
S \partial_{1}=\partial_{2}, \quad S \partial_{2}=0,
$$

and $g\left(\partial_{1}, \partial_{1}\right)=1, g\left(\partial_{1}, \partial_{2}\right)=\varepsilon$ where $\varepsilon \in\{-1,1\}$. The frame $\left\{\partial_{1}, \partial_{2}\right\}$ is $\nabla^{\wedge}$-parallel.

Proof (we follow [1]). Let $X$ be a local field on $M$ satisfying

$$
g(X, X)=1, \quad g(X, S X)=\varepsilon .
$$

Then $X$ and $S X$ are linearly independent. From (10) we obtain

$$
g\left(\nabla_{Y}^{\wedge} X, X\right)=0, \quad g\left(\nabla_{Y}^{\wedge} X, S X\right)=0,
$$

which follows from $\nabla^{\wedge} S=0, \nabla^{\wedge} g=0$ and from (R). This means $\nabla^{\wedge} X=0$ and also $\nabla^{\wedge} S X=0$ as $\nabla^{\wedge} S=0$. Take

$$
\partial_{1}=X, \quad \partial_{2}=S X .
$$

Then

$$
\left[\partial_{1}, \partial_{2}\right]=\nabla_{\partial_{1}}^{\wedge} \partial_{2}-\nabla_{\partial_{2}}^{\wedge} \partial_{1}=0
$$

which means that there exists a chart $\left(U, x^{1}, x^{2}\right)$ on $M$ such that $\partial_{i}=\partial / \partial x^{i}$. From (12) it also follows that $\nabla^{\wedge} \partial_{i}=0$.

Proposition 2. Let $(M, f)$ be a nondegenerate hypersurface in $\mathbb{R}^{3}$ with an equiaffine structure $(\nabla, \xi)$ and a parallel shape operator, which is not a sphere. Then $\nabla R=0$ (i.e. $\nabla$ is locally symmetric) if and only if $(\nabla, c \xi)$ is a Blaschke structure for a certain nonzero $c \in \mathbb{R}$.

Pro of. Note that in our case the equation

$$
K\left(X, \partial_{2}\right)=0
$$

is equivalent to $\nabla R=0$. Indeed, from (4) and $\nabla S=0$ it follows that

$$
\nabla R(W, X, Y) Z=\nabla g(W, Y, Z) S X-\nabla g(W, X, Z) S Y .
$$

We have to show that $\nabla R\left(W, \partial_{1}, \partial_{2}, Z\right)=0$ for all $W, Z$, which by (S1) is equivalent to

$$
\nabla g\left(W, \partial_{2}, Z\right) \partial_{2}=0
$$

By (3) this is equivalent to $g\left(W, K\left(\partial_{2}, Z\right)\right)=0$. Hence $\nabla R=0$ is equivalent to $(\mathrm{K})$.

$\Leftarrow$ Let $\left(U, x^{1}, x^{2}\right)$ be a chart on $M$ as in the Lemma. The apolarity condition (A) in our case has the form (as $g^{11}=0, g^{12}=\varepsilon, g^{22}=-1$ )

$$
2 \varepsilon C\left(X, \partial_{1}, \partial_{2}\right)-C\left(X, \partial_{2}, \partial_{2}\right)=0 \text {. }
$$


From Proposition 1 using (3) we get $K\left(\partial_{2}, \partial_{2}\right)=S^{2} K\left(\partial_{1}, \partial_{1}\right)=0$, hence $C\left(X, \partial_{2}, \partial_{2}\right)=0$. From (A1) we obtain

$$
C\left(X, \partial_{1}, \partial_{2}\right)=-2 g\left(X, K\left(\partial_{1}, \partial_{2}\right)\right)=0,
$$

hence $K_{\partial_{2}}=0$, which implies $\nabla R=0$.

$\Rightarrow \nabla R=0$ implies $(\mathrm{K})$, hence $K_{\partial_{2}}=0$, by virtue of (3) yielding the apolarity condition (A1); hence $\nabla$ is a Blaschke connection.

THEOREM 2. Let $(M, f)$ be a nondegenerate hypersurface in $\mathbb{R}^{3}$ with an equiaffine structure $(\nabla, \xi)$ such that $\nabla S=0$ and $\nabla R=0$. Then one of the conditions below holds:

(a) $(M, f)$ is a nondegenerate quadratic surface.

(b) $(M, f)$ is affinely equivalent to one of the surfaces $\left(\mathbb{R}^{2}, f_{i, \phi}\right)$.

(c) $S=0$, i.e. $(M, f)$ is an improper equiaffine sphere.

Proof. From the Lemma, (2) and (K) we have $\nabla_{\partial_{2}} \partial_{2}=\nabla_{\partial_{1}} \partial_{2}=0$, $\nabla_{\partial_{1}} \partial_{1}=\Gamma_{11}^{2} \partial_{2}$ as $S\left(\nabla_{\partial_{1}} \partial_{1}\right)=\nabla_{\partial_{1}} \partial_{2}=0$. Hence

$$
\varepsilon \Gamma_{11}^{2}=g\left(\nabla_{\partial_{1}} \partial_{1}, \partial_{1}\right)=-(1 / 2) \nabla g\left(\partial_{1}, \partial_{1}, \partial_{1}\right)
$$

in view of (3) as $\nabla_{\partial_{1}} \partial_{1}=K\left(\partial_{1}, \partial_{1}\right)$. This implies that

$$
\partial_{2} \Gamma_{11}^{2}=\varepsilon \nabla g\left(\partial_{2}, \nabla_{\partial_{1}} \partial_{1}, \partial_{1}\right)+\varepsilon g\left(\nabla_{\partial_{2}} \nabla_{\partial_{1}} \partial_{1}, \partial_{1}\right) .
$$

But (K) and (3) imply

$$
\nabla g\left(\partial_{2}, \nabla_{\partial_{1}} \partial_{1}, \partial_{1}\right)=-2 g\left(K\left(\partial_{2}, \partial_{1}\right), \nabla_{\partial_{1}} \partial_{1}\right)=0
$$

and we also have

$$
\nabla_{\partial_{2}} \nabla_{\partial_{1}} \partial_{1}=R\left(\partial_{2}, \partial_{1}\right) \partial_{1}
$$

It follows from (14) that

$$
\begin{aligned}
\partial_{2} \Gamma_{11}^{2} & =\varepsilon g\left(R\left(\partial_{2}, \partial_{1}\right) \partial_{1}, \partial_{1}\right)=\varepsilon g\left(-g\left(\partial_{2}, \partial_{1}\right) S \partial_{1}, \partial_{1}\right) \\
& =-\varepsilon g\left(\partial_{2}, \partial_{1}\right) g\left(\partial_{2}, \partial_{1}\right)=-\varepsilon
\end{aligned}
$$

This implies that

$$
\Gamma_{11}^{2}=-\varepsilon y^{2}+\phi\left(y^{1}\right)
$$

for a certain $\phi \in C^{\infty}(\mathbb{R})$. Hence the immersion $f \circ \psi^{-1}$, where $\psi(p)=$ $\left(y^{1}(p), y^{2}(p)\right)$ and $f_{1, \phi}$ when $\varepsilon=1$ or $f_{2, \phi}$ in the case $\varepsilon=-1$ define the same structure on $\psi(U)$, i.e.

$$
g=g_{i, \phi}, \quad \nabla=\nabla_{i, \phi} .
$$

Our theorem is then a consequence of the following theorem (see [2]).

Theorem. Let $\left(M, f_{i}\right)$ be two nondegenerate hypersurfaces in $\mathbb{R}^{n+1}$ with equiaffine structures $\left(\nabla_{i}, \xi_{i}\right)$ such that $g_{1}=g_{2}, \nabla_{1}=\nabla_{2}$. Then there exists an affine isomorphism $A \in \operatorname{AGL}(n+1)$ such that $A f_{1}=f_{2}$. 
Remark. From the proof of Theorem 2 it is clear that $\left(\mathbb{R}^{2}, f_{i, \phi}\right)$ is affinely equivalent to $\left(\mathbb{R}^{2}, f_{i, \psi}\right)$ if and only if $\phi(x)=\gamma \psi(\gamma x+a)+b$ where $\gamma= \pm 1$ and $a, b \in \mathbb{R}$ (see (13)).

Acknowledgements. The author wishes to express his hearty thanks to Prof. L. Vrancken for his valuable advice.

\section{References}

[1] F. Dillen, Locally symmetric complex affine hypersurfaces, J. Geom. 33 (1988), 2738.

[2] -, Equivalence theorems in affine differential geometry, Geom. Dedicata 32 (1989), $81-92$.

[3] M. Magid and K. Nomizu, On affine surfaces whose cubic forms are parallel relative to the affine metric, Proc. Nat. Acad. Sci. U.S.A. 65 (1989), 215-218.

[4] K. Nomizu, On completeness in affine differential geometry, Geom. Dedicata 20 (1986), 43-49.

[5] K. Nomizu and U. Pinkall, On the geometry of affine immersions, Math. Z. 195 (1987), 165-178

[6] - - - Cayley surfaces in affine differential geometry, Tôhoku Math. J. 41 (1989), 589-596.

INSTITUTE OF MATHEMATICS

TECHNICAL UNIVERSITY OF CRACOW

WARSZAWSKA 24

31-155 KRAKÓW, POLAND

Reçu par la Rédaction le 21.11.1990

Révisé le 26.3.1991 